\title{
4C39.25 - SUPERLUMINAL MOTION BETWEEN STATIONARY COMPONENTS
}

\author{
D. B. Shaffer \\ Interferometrics Inc. \\ 8150 Leesburg Pike \\ Vienna, Virginia 22180 USA \\ and
}

A. P. Marscher

Department of Astronomy

Boston University

725 Commonwealth Avenue

Boston, Massachusetts 02215 USA

\begin{abstract}
During the 1970's, the compact radio structure of $4 \mathrm{C} 39.25$ consisted of a simple double source, whose separation did not change. A new component, which probably originated in mid-1979, is now seen to be moving superluminally between the original, still stationary, comporents. It is not clear which component of the source is the core, nor how the moving component will interact with the stationary component that it is now approaching on a collision course.
\end{abstract}

From 1972 until 1979, the compact radio structure of the quasar 4C39.25 $(\mathrm{z}=0.699)$ consisted of an almost east/west (position angle $98^{\circ}$ ) double source, whose separation was about 2.0 mas at frequencies from 5 to $15 \mathrm{GHz}$ (Shaffer et al. 1977 and 1987). In late 1979, the structure of the westernmost component of the source began to change, and by 1982 a new component was clearly evident between the two original components. Since then, the source structure has consisted of three distinct components. The central component is moving eastward with an angular velocity of $0.16 \pm 0.02$ mas year $^{-1}$ with respect to the two outer components, which have the original 2.0 mas separation. The corresponding velocity is $3.5 h^{-1} c$ for $q_{0}=0.5$ and $H_{0}=100 h \mathrm{~km} \mathrm{~s}^{-1} \mathrm{Mpc}^{-1}$. The epoch of zero separation of the central component from the westernmost component of the original double is about 1979.5. This time coincides with a plateau in the long-term decline of the flux density of the source (Aller et al. 1985), perhaps indicative of a weak outburst.

Shaffer et al. (1987) show maps of the structure of $4 \mathrm{C} 39.25$ at 8.4 and $10.7 \mathrm{GHz}$, from 1979 through 1985 . Our 1986 maps at $8.4 \mathrm{GHz}$ show that the motion of the central component has continued at the same rate. Observations at $22 \mathrm{GHz}$ (Marscher et al. 1987) also show a triple source, whose central component is the brightest and most compact.

The structure and its variations in 4C39.25 are quite unlike those seen in any other source, and it is not clear how to interpret these observations. For instance, we are not certain which component is the nucleus of the source. With no other information, one would be inclined to call the central component the core because it is so strong and compact at $1.3 \mathrm{~cm}$. Such characteristics usually identify the nuclear component. Alternatively, since the central component is seen to be moving to the east, away from the western component, one would argue that the western component is the core, by analogy to all other superluminal sources, since that is where the moving component seems to have originated. 
On the arcsecond scale, 4C39.25 shows a two-sided jet, with the brightest component on the east (Browne et al. 1982). In almost all other sources, the superluminal components move out towards the brightest outer features ( $3 \mathrm{C} 120$ or $3 \mathrm{C} 273$, for example). If $4 \mathrm{C} 39.25$ is similar, its extended structure is consistent with the western component being the core. The source clearly has had two-sided ejection in the past, but we can not tell if we are seeing only one-sided activity now or just the beamed side of a two-sided jet.

We can not even tell for sure which component(s) is (are) really moving. We do not have any strong limits on the overall motion of the source, except that if its position had changed by more than about one or two milliarcseconds over the last five years, we would have detected the motion with NASA geodesy observations and analysis. It is possible that the central component is stationary, and that the two outer components are moving with the same velocity, or that all three components are moving but with different velocities.

Both the outer components could be stationary features in a relativistic jet. Depending on its environment, such a jet can alternately expand and contract, showing compact hot spots where the contraction reaches a local maximum. In this case, the central component would then be a shock or instability caught up in the flow.

It is possible, although we feel it is unlikely, that the outer components are gravitational lens images of the same compact core. In this case, both "cores" should be seen to eject the same component, although not necessarily at the same time because of path length differences for the two images. There is no evidence for lensing in the arcsecond structure of the source (Browne et al.).

Which, if any, of these models is correct may be revealed as we follow the evolution of 4C39.25. The central component is on a collision course with the eastern component. What happens when (or if) a collision occurs should provide considerable insight into the nature of radio jets and the composition of superluminal material. The actual encounter, due in 1991 at the present pace, may show one of several possible results: If the eastern component is a stationary, compact lobe being fed by the jet or caused by an obstruction in the jet flow, the moving component should stop when it gets there, and the eastern end of the source will probably brighten. If the eastern component is a contraction in an undulating jet, the moving component should pass through it at constant velocity, although there may be a brightening as the moving material is compressed. If the components are just images, they should show no interaction at all. We are continuing our observations of this fascinating object, waiting to see what happens.

\section{REFERENCES}

Aller, H. D., Aller, M. F., Latimer, G. E., and Hodge, P. E. 1985, Ap. J. Suppl., 59, 513.

Browne, I. W. A., Clark, R. R., Moore, P. K., Muxlow, T. W. B., Wilkinson, P. N., Cohen, M. H., and Porcas, R. W. 1982, Nature, 299, 788.

Marscher, A. P., Shaffer, D. B., Booth, R. S., and Geldzahler, B. J. 1987, Ap. J. Letters, submitted.

Shaffer, D. B. et al., 1977, Ap. J., 218, 353.

Shaffer, D. B., Marscher, A. P., Marcaide, J., and Romney, J. D. 1987, Ap. J. Letters, 314, L1. 